\title{
Laser Beam Profile Influence on Dark Hanle Resonances in $\mathrm{Rb}$ Vapor
}

\author{
A.J. Krmpot*, S.M. Ćuk, S.N. Nikolić, M. Radonjić, Z.D. Grujić and B.M. Jelenković \\ Institute of Physics, Belgrade, Serbia
}

\begin{abstract}
Influence of two different laser beam profiles, the Gaussian and the $\Pi$ (top hat) profile on the resonance line widths and amplitudes in the Hanle electromagnetically induced transparency was studied. The laser beam propagates through the vacuum $\mathrm{Rb}$ glass cell. Studies were done at $D_{1}$ line for the open ${ }^{87} \mathrm{Rb}: F_{\mathrm{g}}=2 \rightarrow F_{\mathrm{e}}=1$ transition. Hanle electromagnetically induced transparency was measured for the two beam profiles with the same total power and beam diameter and experimental results showed that Gaussian and the top hat profiles give different amplitudes and widths of the Hanle resonances. Resonances obtained from the top hat laser beam profile have lower amplitudes and higher line widths.
\end{abstract}

PACS numbers: $32.70 . \mathrm{Jz}, 42.50 . \mathrm{Gy}, 42.62 . \mathrm{Fi}, 32.30 . \mathrm{Jc}$

\section{Introduction}

Coherent effects in the Doppler broadened alkali atom vapor have been intensively investigated over the past decade. Coherent population trapping (CPT) [1, 2], electromagnetically induced transparency (EIT) [3], and electromagnetically induced absorption (EIA) $[4,5]$ have been observed and examined in pump-probe and in the Hanle configuration [6]. All these effects nonlinearly depend on intensity of the applied laser field. Several papers have theoretically studied the dependence of coherent resonances on laser intensity [7-9], but most assume top hat laser beam profile due to a simplified theoretical analysis. The influence of other beam profiles, such as experimentally accessible Gaussian profile, on CPT line shape has been described only in a few papers [10-12], but there are no experimental studies dealing with the influence of the laser beam profile on the EIT line shapes.

The aim of this paper is to experimentally represent difference in line widths and amplitudes of Hanle EIT resonances for the two laser beam profiles, the Gaussian and the top hat one. Our investigation was performed on ${ }^{87} \mathrm{Rb}$ atoms at $D_{1}$ line in the vacuum cell. We presented notably different Hanle EIT resonances related to the Gaussian and the top hat profiles of the laser beam. Comparing our results with those given in [11] the significant differences between dependence of dark resonances on the laser intensity for vacuum and buffer gas cell could be observed.

\section{Experiment}

The experimental setup is shown in Fig. 1. External cavity diode laser is frequency locked to $F_{\mathrm{g}}=2 \rightarrow F_{\mathrm{e}}=1$

\footnotetext{
* corresponding author; e-mail: krmpot@phy.bg.ac.yu
}

transition in ${ }^{87} \mathrm{Rb}$ by Doppler-free dichroic atomic vapor laser lock (DDAVLL) method [13] in the vacuum $\mathrm{Rb}$ cell. The variable neutral density filter is used for the laser power adjustments. After passing through the Glen-Thomson polarizer the laser beam becomes linearly polarized. The Gaussian beam profile with $3 \mathrm{~mm}$ in diameter is obtained by expanding the beam exiting the single mode fiber. For the definition of the laser beam radius we use the distance from the beam maximum where the maximal intensity drops $\mathrm{e}^{2}$ times. For the top hat profile the Gaussian beam is further expanded to $20 \mathrm{~mm}$ and sent through the $3 \mathrm{~mm}$ aperture to extract the central part of the laser beam.

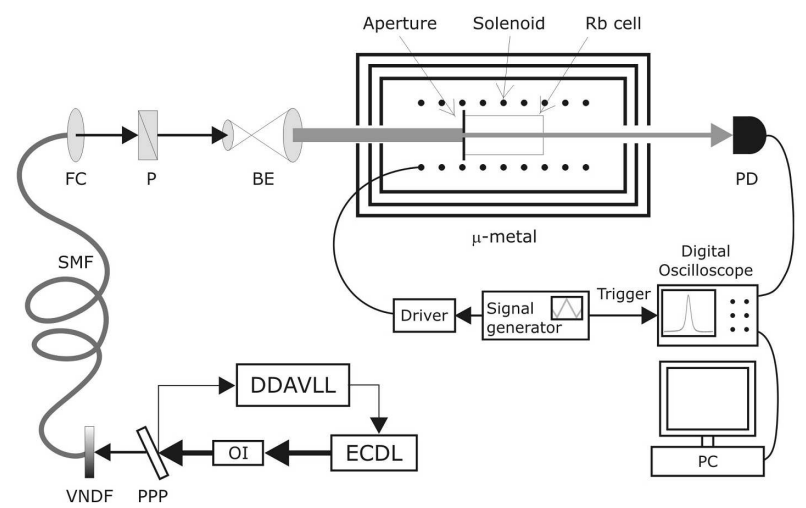

Fig. 1. Experimental setup. ECDL - external cavity diode laser, OI - optical isolator, DDAVLL — Doppler-free dichroic atomic vapor laser lock, VNDF - variable neutral density filter, SMF - single mode fiber, FC fiber collimator, $\mathrm{P}$ - polarizer, BE - beam expander, $\mathrm{PD}$ - photodiode.

The laser beam, either Gaussian or top hat profile, passes through $5 \mathrm{~cm}$ long vacuum $\mathrm{Rb}$ cell containing nat- 
ural abundance of rubidium isotopes. The cell is placed in the solenoid used for scanning the axial magnetic field between $\pm 3 \mathrm{G}$. The cell and the solenoid are inside the triple layered $\mu$-metal cylinder in order to eliminate Earth's and stray magnetic fields.

The signal obtained from the photodiode, while scanning the external magnetic field, is recorded by the digital oscilloscope and transferred to the computer.

\section{Results and discussion}

We analyze the Hanle line shapes for the two laser beam profiles. Both the Gaussian and the top hat laser beams have the same total power $P$ and the beam diameter $d$. The dependence of the intensity on the transverse distance $r$ to the beam center can be written as

$$
I(r)=I_{0} f\left(r / r_{0}\right)
$$

where the $I_{0}$ is the maximal intensity corresponding to $r=0, r_{0}$ is the radius of the beam $\left(r_{0}=d / 2\right)$, and $f\left(r / r_{0}\right)$ is a profile function $\left(0<f\left(r / r_{0}\right)<1\right)$. Thus, for the top hat profile we have

$$
f\left(r / r_{0}\right)= \begin{cases}1, & r \leq r_{0}, \\ 0, & r \geq r_{0},\end{cases}
$$

and for the Gaussian profile

$$
f\left(r / r_{0}\right)=\exp \left(-2\left(r / r_{0}\right)^{2}\right) \text {. }
$$

The Hanle EIT resonances for Gaussian and top hat laser beam profiles, at the laser intensity $1.8 \mathrm{~mW} / \mathrm{cm}^{2}$, are shown in Fig. 2. As could be seen, the resonance shape becomes sharper for the Gaussian profile compared to the top hat intensity distribution. This results in slightly narrower line in the case of the Gaussian beam.

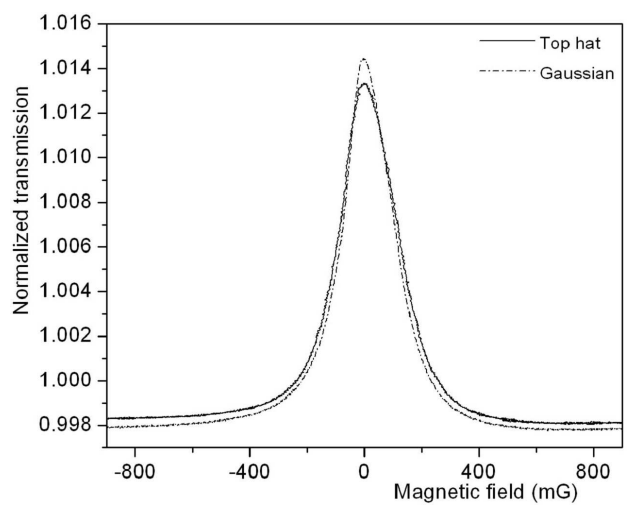

Fig. 2. Hanle EIT resonances obtained for two different laser beam profiles: Gaussian (dash dot) and top hat (solid). The beam diameter was $d=3 \mathrm{~mm}$ and the total laser intensity was $I=1.8 \mathrm{~mW} / \mathrm{cm}^{2}$.

In Fig. 3, the dependence of the Hanle EIT resonance amplitude (a) and line width (b) on the laser beam intensity is shown. According to our experimental results, for the cases of top hat (square symbol) and Gaussian (open triangle symbol) laser beam profile, the intensity dependence of resonance amplitude is nonlinear, and the amplitude is higher for the Gaussian profile in comparison with the top hat. The amplitudes of the Hanle EIT resonances for Gaussian beam could be higher up to $12 \%$ than those for top hat profile, in the given range of laser intensities. Intensity dependences of line widths are monotonically increasing functions, with very different slopes; the EIT line widths obtained using the top hat laser beam profile are becoming wider than for the Gaussian beam up to the $18.5 \%$, as the laser intensity increases.
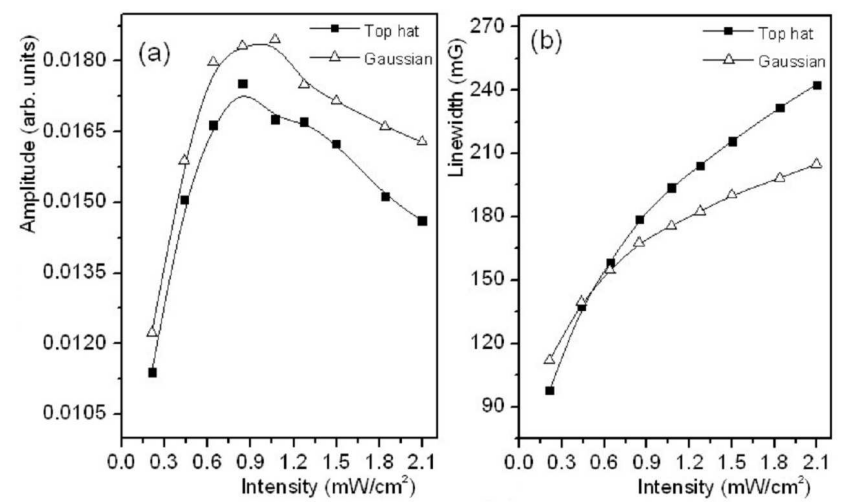

Fig. 3. Amplitude (a) and line width (b) of the Hanle EIT resonances versus laser intensity given for the Gaussian (open triangle symbol) and for the top hat laser beam profile (square symbol).

The atom experiences different excitations when enters different radial profiles of the laser intensity. For the Gaussian beam, the intensity reaches its maximal value $I_{0}$ only at the very center of the beam, while for the top hat profile this peak intensity is present all over the beam. Therefore, in the top hat beam profile all atoms see the same intensity, whereas for the Gaussian beam atoms in the outer regions are experiencing much lower laser intensity, contributing with narrower lines to the overall line shape. Top hat beam profile has significantly faster increase of light intensity at its edge, so that an atom that enters the laser beam will be promoted faster to the dark state in comparison with Gaussian profile. It is now obvious why the profile function is important when analyzing experimental dependences. However, in practice one usually calculates the field intensity as $P / S$, with $S$ being the area of the light spot, without a detailed investigation of the intensity profile. Thus, for seemingly identical parameters, different experimental setups can produce different results.

When comparing our experimental results with theoretical predictions for buffer gas cell given in [11], we observe similarity in behavior of line widths versus laser intensity, i.e. Gaussian beam profile results in narrower EIT resonances than top hat profile. From Fig. 3b it could be seen that previous statement is not valid only at extremely low intensities. Concerning amplitudes of EIT lines, situation in vacuum cell is significantly different from that in a buffer gas cell. Namely, in the case of a vacuum cell Gaussian beam profile gives, particularly at higher laser intensities, higher amplitudes than top 
hat profile. Situation in a buffer gas cell is vice versa, the dependence is more or less linear and Gaussian beam profile results in less intense CPT lines than top hat, over all intensities.

\section{Conclusion}

This study has shown that different laser beam profiles considerably influence the Hanle EIT line profiles. Assuming the same laser power and diameter for the two beam profiles, the resonances obtained from the Gaussian laser beam are narrower than those obtained with the top hat profile, particularly at higher laser intensities. This is similar to the findings in the buffered gas cells. Contrary to the results with buffered gas cells, we have found that in the vacuum gas cell amplitudes of EIT resonances are lower for the top hat laser beam profile.

\section{References}

[1] E. Arimondo, Prog. Opt. 35, 257 (1996).

[2] G. Alzetta, A. Gozzini, L. Moi, G. Orriolis, Il Nuovo Cimento 36, 5 (1976).

[3] S.E. Harris, J.E. Field, A. Imamoglu, Phys. Rev. Lett. 64, 1107 (1990).
[4] A.M. Akulshin, S. Barreiro, A. Lezama, Phys. Rev. A 57, 2996 (1998).

[5] G. Moruzzi, F. Strumia, The Hanle effect and Level Crossing Spectroscopy, Plenum Press, 1991.

[6] C. Andreeva, S. Cartaleva, Y. Dancheva, V. Biancalana, A. Burchianti, C. Marinelli, E. Mariotti, L. Moi, K. Nasyrov, Phys. Rev. A 66, 502 (2002).

[7] A. Javan, O. Kocharovskaya, H. Lee, M.O. Scully, Phys. Rev. A 66, 013805 (2002).

[8] C.Y. Ye, A.S. Zibrov, Phys. Rev. A 65, 023806 (2002).

[9] A.J. Krmpot, M.M. Mijailović, B.M. Panić, D.V. Lukić, A.G. Kovačević, D.V. Pantelić, B.M. Jelenković, Opt. Express 13, 1448 (2005).

[10] F. Levi, A. Godone, J. Vanier, S. Micalizio, G. Modugno, Eur. Phys. J. D 12, 53 (2000).

[11] M.A.V. Taichenachev, A.M. Tumaikin, V.I. Yudin, M. Stahler, R. Wynands, J. Kitching, L. Hollberg, Phys. Rev. A 69, 024501 (2004).

[12] M. Radonjić, D. Arsenović, Z. Grujić, B.M. Jelenković, Phys. Rev. A 79, 023805 (2009).

[13] G. Wasik, W. Gawlik, J. Zachorowski, W. Zawadzki, Appl. Phys. B 75, 613 (2002). 